\title{
Pediatric Respiratory Therapists Lack a Standard Mental Model for Managing the Patient Who Is Difficult to Ventilate: A Video Review
}

\author{
Julianne S Perretta, Cheryl Salamone, Daenna King, Stacey Mann, Jordan Duval-Arnould, \\ and Elizabeth A Hunt
}

\begin{abstract}
BACKGROUND: All health-care providers who care for infants and children should be able to effectively provide ventilation with a bag and a mask. Respiratory therapists (RTs'), as part of rapid response teams, need to quickly identify the need for airway support and use adjunct airway interventions when subjects are difficult to mask ventilate. Before implementation of an educational curriculum for airway management, we assessed whether pediatric RTs' who enter the room of a simulated infant mannequin in severe respiratory distress are able to apply bag-mask ventilation within $60 \mathrm{~s}$ and implement 2 adjunct airway maneuvers in a patient who is difficult to ventilate. METHODS: All pediatric RTs' were required to attend one high-fidelity simulation at the Johns Hopkins Medicine Simulation Center. The sessions were reviewed to evaluate whether the therapists would implement adjunct maneuvers to a patient who was in respiratory distress and was difficult to ventilate. RESULTS: Twenty-eight therapists participated in the baseline skills assessment session, and 26 (72\% of eligible therapists) were evaluable with video clips. Only 3 of 26 (12\%) attempted bag-mask ventilation within $60 \mathrm{~s}$. Although all the therapists attempted one airway maneuver, only $65 \%$ were able to implement $\geq 2$ airway maneuvers and achieve effective ventilation, with a wide range of time (98-298 s). There was no pattern regarding which intervention was implemented first, second, and so forth. CONCLUSIONS: Our team of pediatric RTs' did not share a standard mental model for initiating bag-mask ventilation during impending respiratory failure or implementing airway adjuncts. This may place children who are critically ill at risk of suboptimal management and threaten clinical outcomes. Therapist performance indicated that no established care algorithm had been effectively implemented or that skill retention was poor. A change in the content and delivery method of bag-mask ventilation training is warranted to improve the time to performance of key interventions and to establish a clear cognitive framework of difficult mask ventilation management. Key words: respiratory therapy; bag-mask ventilation; high-fidelity simulation; educational curriculum; difficult mask ventilation; airway assessment. [Respir Care 2019;64(7):801-808. (C) 2019 Daedalus Enterprises]
\end{abstract}

\section{Introduction}

The American Heart Association and American Academy of Pediatrics posit that all health-care providers who care for infants and children should be able to effectively provide ventilation with a bag and a mask. ${ }^{1}$ This interven-

\footnotetext{
Mrs Perretta, Dr Duval-Arnould, and Dr Hunt are affliated with The Johns Hopkins Medicine Simulation Center, The Johns Hopkins University, Department of Pediatric Anesthesiology and Critical Care Medicine, Baltimore, Maryland. Mrs Salamone, King, and Mann are affliated with The Johns Hopkins Hospital, Division of Pediatric Respiratory Care Services, Baltimore, Maryland.
}

tion requires a distinct set of skills to implement effectively, which includes selecting and preparing the correct equipment, positioning the patient, and correctly position-

\footnotetext{
Dr Hunt discloses a relationship with Zoll Medical. Dr Duval-Arnould discloses a relationship with Zoll Medical. The remaining authors have disclosed no conflicts of interest.

Correspondence: Julianne S Perretta MSEd, RRT-NPS, CHSE-A, The Johns Hopkins Medicine Simulation Center, $601 \mathrm{~N}$ Caroline St, Suite 8210 Baltimore, MD 21287. E-mail: jperret1@jhmi.edu.
}

DOI: $10.4187 /$ respcare.06369 


\section{RTs' Response Time to Simulated Difficult Bag-Mask Ventilation}

ing the clinician who provides bag-mask ventilation. A review of epidemiologic data from our institution revealed 120 pediatric rapid response team calls for primary respiratory events in 2017, which demonstrated that assistive ventilation is an essential skill in this patient population. Informal observation during pediatric rapid responses indicates that there is a broad variation in the competence level of this fundamental skill by all levels of providers.

Because pediatric subjects have higher basal oxygen consumption and lower pulmonary reserve than adults, they develop hypoxemia much faster if their ventilation is compromised. ${ }^{2}$ These subjects require rapid intervention to stabilize the airway and provide bag-mask ventilation to prevent hypoxemic neurologic injury and/or progression to cardiac arrest. Unfortunately, the relatively large occiput, redundant soft tissue, and a larger tongue of young children can make bag-mask ventilation difficult. ${ }^{3}$ In fact, despite effective initial skills, $6.6 \%$ of otherwise healthy children will be difficult to bag-mask ventilate. ${ }^{4}$ There is no standard definition for difficult mask ventilation, but a reasonable suggested definition is "inadequate ventilation evidenced by no perceptible chest movement, oxygen desaturation, and perception of severe gas flow leak around the mask."5 Another definition includes the inability of one airway provider to provide adequate mask ventilation as evidenced by "absent or inadequate chest movement, absent or inadequate breath sounds, auscultatory signs of severe obstruction, cyanosis, gastric air entry or dilatation, decreasing or inadequate oxygen saturation, absent or inadequate exhaled carbon dioxide . . . and hemodynamic changes associated with hypoxemia or hypercarbia."6 Management of difficult mask ventilation is a dynamic process in which close observation of ventilation effectiveness should be simultaneously accompanied by modifications in the maneuvers, the use of adjuncts, and a call for help as soon as it seems to be needed. ${ }^{7}$

A review of standard airway management curriculum shows a lack of standardization when teaching which steps to take (and in which order) when initial bag-mask ventilation is ineffective. ${ }^{1,8-12}$ A standard mental model provides a cognitive framework or representation of potential real-life situations, which allows providers to anticipate events, formulate plans, and apply in times of need, such as processing clinical assessment and implementing critical interventions. ${ }^{8}$ The lack of a standard mental model can make it difficult for team members to use the same method to approach this clinical emergency. Before the introduction of a new educational curriculum, we wanted to assess whether pediatric RTs' called to a simulated infant in severe respiratory distress were able to do the following: apply bag-mask ventilation in an attempt to move the chest within $60 \mathrm{~s}$ of entering the room and demonstrate at least 2 adjunct airway maneuvers when his or her initial bag-mask ventilation attempts did not result in

\section{QUICK LOOK}

\section{Current knowledge}

Difficult mask ventilation is an essential skill for clinicians who respond to subjects with impending respiratory failure. There is no standard definition, identified response time, or established algorithm for difficult mask ventilation management to guide clinicians in this highrisk, time-sensitive intervention.

\section{What this paper contributes to our knowledge}

We reviewed pediatric RTs' responses to a simulated infant in impending respiratory failure who was difficult to mask ventilate. We noted a wide range of times to initiate bag-mask ventilation and no pattern in selecting airway adjuncts, or in what order they were implemented. Analysis indicated that no established mental model had been effectively provided or that skill retention was poor. A change to the content and delivery method of difficult airway management training is warranted to improve clinical performance.

visible chest rise. Results of this data collection will help guide the further design of a pediatric difficult mask ventilation curriculum.

\section{Methods}

All pediatric RTs' were required, as part of their annual skills training, to attend one high-fidelity simulation at the Johns Hopkins Medicine Simulation Center. Sessions were recorded by using the SimCapture software program (BLine Medical Corporation, Washington, DC) for quality assurance purposes. When selecting camera angles, care was taken to allow maximum visualization of airway management, while avoiding angles that would allow viewing of the therapist's face, to minimize identification of each subject by the reviewers. A SimBaby (Laerdal Medical, Wappinger Falls, NY) was prepared supine in a hospital crib with pharyngeal obstruction, tongue edema, cyanosis, and audible grunting. RTs' completed the scenario individually; they were told that they had just been called to the infant in-patient unit as a member of the rapid response team; when they arrived in the room, the patient's bedside nurse (played by an embedded actor) provided a verbal report on the patient's current status and recent medical history.

On arrival, the vital signs displayed on the monitor were as follows: heart rate, 110 beats/min; breathing frequency, 8 breaths/min; and $\mathrm{S}_{\mathrm{pO}_{2}} 85 \%$. All other vital signs were within normal limits. All equipment that would be 


\section{RTs' Response Time to Simulated Difficult Bag-Mask Ventilation}

available at the patient's bedside was available to the RT, including an infant resuscitation bag and 2 mask sizes. The RT was given the emergency respiratory care bag and was given the option to use anything within the bag to care for the infant. RTs' bring this bag, which includes all available sizes of laryngeal mask airways, intubation supplies, oral and nasal airways, and inhalation therapy equipment, to all rapid response calls. On entry into the room, the simulated patient's vital signs were pre-programmed to deteriorate over a 2-min period to a heart rate of 80 beats/ min and $\mathrm{S}_{\mathrm{pO}_{2}}$ of $75 \%$, Once bag-mask ventilation with 2 interventions was provided by the RT, the patient's vital signs stabilized, including resolution of cyanosis, reversal of tongue edema and pharyngeal obstruction (to provide effective chest rise), and $\mathrm{S}_{\mathrm{pO}_{2}}$ increase to $92 \%$. Correct adjunct airway interventions included the items listed as follows:

- Request 2-person bag-mask ventilation

- Incorporate shoulder or head roll to facilitate sniffing position

- Insert oral airway

- Insert nasal airway

Of note, repositioning the airway is considered a basic airway management skill and thus was not considered an adjunct airway intervention during video review. The simulation concluded when bag-mask ventilation with 2 interventions was completed or when additional members of the rapid response team arrived in the room and intubation preparation commenced, which occurred between minute 4 and minute 5. A diagram that illustrates the scenario flow and key points in data collection are illustrated in Figure 1.

The facilitators debriefed each RT immediately on conclusion of the simulation by using advocacy and inquiry for observed patient assessment and decision-making performance gaps and directed debriefing for skills-based performance gaps. When all staff sessions were complete, the videos were reviewed by 2 advanced-level pediatric RTs' [CS and DK] by using a checklist of key performance measures. A subset of 9 videos was assessed by both reviewers independently to verify inter-rater reliability, which was assessed by using Cohen's kappa. All critical time measurements (time zero, time to initiate bag-mask ventilation, and bag-mask ventilation after 2 adjunct interventions) were obtained by an additional researcher [JP] to verify the results. The participants were oriented to the video recording device such that facial features were not captured during the session. A significant degree of participant anonymity was achieved as a result, and the ability of the reviewer to identify the participant was limited. Video review was approved by our internal institutional review board, and the subjects consented per institutional review board requirements.

The primary outcome measure was the time to apply 2 adjunct airway maneuvers and reapply bag-mask ventilation. We were particularly interested to see which interventions were selected and whether there were any common themes related to the order of the interventions. We also reviewed videos to evaluate how quickly RTs' applied bag-mask ventilation to a patient in respiratory distress, to see whether timely initiation of assistive ventilation was a necessary future educational intervention.

\section{Results}

The annual skills training was held over the course of 3 months, and a convenience sample of 26 therapists' performance videos were reviewed. This represented $72 \%$ of eligible participants (26/36) (2 others participated but the videos were not of good quality). The clinical experience of the participants ranged from $<1$ y to $26 \mathrm{y}$ (Table 1); the median experience level was $4.5 \mathrm{y}$. All the participants had current American Heart Association Basic Life Support certification, and 10 participants (38\%) had current Pediatric Advanced Life Support (PALS) certification. The sample of the videos reviewed was representative in terms of the proportion of the RT staff who were male (male RT staff, 28\% [11/40]; male subjects, $31 \%$ [8/26]). The data were analyzed by using descriptive statistics. Agreement of the observers was found to be excellent ( 0.85 agreement, $95 \% \mathrm{CI}$ and a $93 \%$ overall agreement).

Only 3 therapists (3/26 [12\%]) initiated bag-mask ventilation within $60 \mathrm{~s}$. The time to complete initial bag-mask ventilation for all the therapists ranged from 24 to $307 \mathrm{~s}$, with a median of $123 \mathrm{~s}$ (interquartile range, 87-161 s). Seventeen therapists (17/26 [65\%]) implemented 2 adjunct airway interventions and reapplied bag-mask ventilation during their simulation. The range of elapsed time to reapply bag-mask ventilation after 2 adjunct airway interventions was 98 to $298 \mathrm{~s}$, with a median of $181 \mathrm{~s}$ (interquartile range, 165-209 s). The time distribution for bag-mask ventilation interventions is shown in Figure 2.

Of the participants who initiated bag-mask ventilation within $60 \mathrm{~s}(n=3)$, one had current PALS certification (33\%). When comparing the time to starting bag-mask ventilation by PALS certification status, there was no significant difference between the groups: the median time for starting bag-mask ventilation for therapists with PALS certification was $105 \mathrm{~s}$ (interquartile range, 84-133 s), versus $101 \mathrm{~s}$ (interquartile range, 56-164 s) for therapists without PALS certification $(P=.97)$. When assessing successful bag-mask ventilation after 2 adjunct airway interventions by PALS certification, we were unable to detect a difference: of those who were successful, $69 \%$ had 
Clinical Simulation Scenario
Infant-Impending Respiratory Failure

SCENARIO DESIGN

$\mathrm{RT}$ is told by instructor that he/she has received a call for a rapid response on the infant care floor

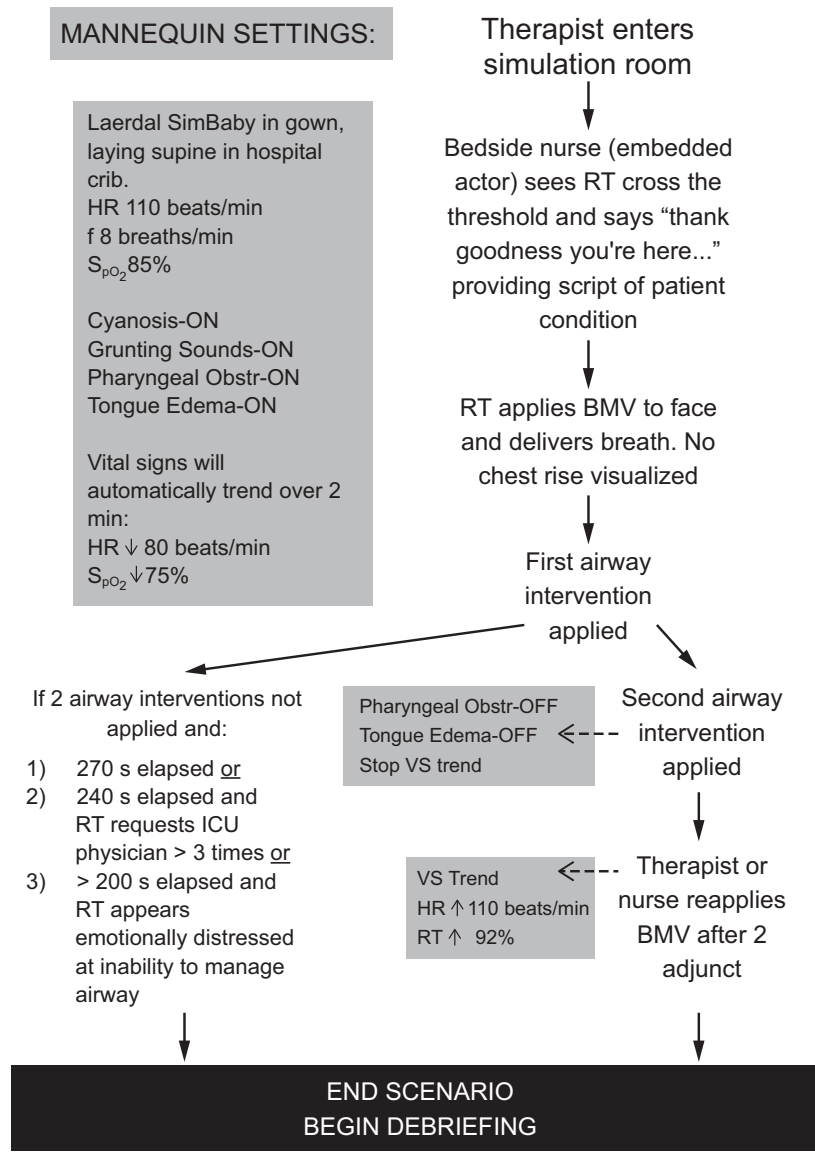

DATA COLLECTION

TIME = ZERO

TIME $=$ BMV

Initiated

Note interventions implemented

TIME = BMV After

2 interventions

END DATA

COLLECTION

Fig. 1. Simulation scenario flow diagram. $R T=$ respiratory therapist, $H R=$ heart rate, $f=$ breathing frequency, $B M V=$ bag-mask ventilation.

current PALS certification versus $60 \%$ did not have current PALS certification $(P=.48)$.

All the therapists attempted at least one intervention when initial bag-mask ventilation was not successful in obtaining chest rise or an increase in $\mathrm{S}_{\mathrm{pO}_{2}}$, but there was no pattern to which intervention was implemented first, second, and so forth (Table 2). For example, 17 of 26 therapists $(65 \%)$ chose to implement an adjunct airway intervention before an initial bag-mask ventilation attempt. The most likely intervention to precede bag-mask ventilation was placement of a shoulder roll; in fact, the same number of therapists in this study chose to place a shoulder roll as their first intervention as those who initiated bag-mask ventilation as their first intervention (10 vs 10$)$.

The number of therapists who chose to implement each of the different airway interventions is illustrated in Figure
3. Oral airway and shoulder roll were the most commonly chosen interventions, with 19 and 14 therapists selecting them, respectively. Three therapists chose to implement 2 adjunct airway maneuvers before initiation of any bagmask ventilation, and 12 therapists did not implement 2 airway interventions despite having at least $200 \mathrm{~s}$ before additional members of the rapid response team arrived in the room. The distribution of time to begin bag-mask ventilation and time to implement bag-mask ventilation after 2 adjunct airway interventions by each RTs' is shown in Figure 4.

\section{Discussion}

In this study, we demonstrated that, in simulated pediatric respiratory failure with difficult mask ventilation, only 


\section{RTs' Response Time to Simulated Difficult Bag-Mask Ventilation}

$65 \%$ of RTs' tried at least 2 adjunctive maneuvers to achieve air movement. El-Orbany and Woehlck ${ }^{7}$ suggested that "mask ventilation is the most basic, yet the most essential, skill in airway management," but we found that it was often minimized or deferred during airway management training in preference for more-invasive maneuvers, for example, intubation skills.

The results of this video review indicated that a morestructured focus on how we teach difficult mask ventila-

Table 1. Demographics of Individual Participants

\begin{tabular}{lr}
\hline \hline \multicolumn{1}{c}{ Characteristic } & $n(\%)$ \\
\hline Sex & \\
$\quad$ Male & $8(31)$ \\
Female & $18(69)$ \\
Experience & \\
$\leq 1$ y & $7(27)$ \\
$2-5$ y & $6(23)$ \\
$5-10$ y & $4(15)$ \\
$10-20$ y & $7(27)$ \\
$\geq 20$ y & $2(8)$ \\
Current Pediatric Advanced Life Support certification & \\
Yes & $10(38)$ \\
No & $16(62)$ \\
Completion of previous annual training with simulation & \\
Yes & $18(69)$ \\
No & $8(31)$ \\
\hline
\end{tabular}

tion management is necessary to improve speed and predictability of providers' responses. This aligns with the results of the study by Kory et al ${ }^{13}$ of senior internal medicine residents, which showed that $2 \mathrm{y}$ of clinical experience was not sufficient to achieve proficiency in initial airway management skills. Our results were consistent with the survey by Eppich et $\mathrm{al}^{8}$ of a mental model for bagmask ventilation, which suggested that there is a lack of a mental model or standardization for interventions by pediatric clinicians when initial ventilation attempts are unsuccessful. Our study expanded on the work by Eppich et $\mathrm{al}^{8}$, which was a written survey that investigated providers' mental models.

Although the study by Eppich et $\mathrm{al}^{8}$ included physician resident trainees from pediatrics, internal medicine, and

Table 2. The Order in Which Interventions Were Implemented

\begin{tabular}{lcccc}
\hline \hline \multirow{2}{*}{ Intervention } & \multicolumn{4}{c}{ Order of Intervention, $n(\%)$} \\
\cline { 2 - 5 } & First & Second & Third & Fourth \\
\hline BMV & $10(38)$ & $7(27)$ & $8(31)$ & $1(4)$ \\
Reposition & $3(12)$ & $9(35)$ & $2(8)$ & 0 \\
2-person BMV & 0 & $2(8)$ & 0 & 0 \\
Shoulder roll & $10(38)$ & $3(12)$ & 0 & $1(4)$ \\
Oral and/or nasal airway & $3(12)$ & $5(19)$ & $14(54)$ & $3(12)$ \\
& & & & \\
BMV = bag-mask ventilation & & & & \\
\hline
\end{tabular}

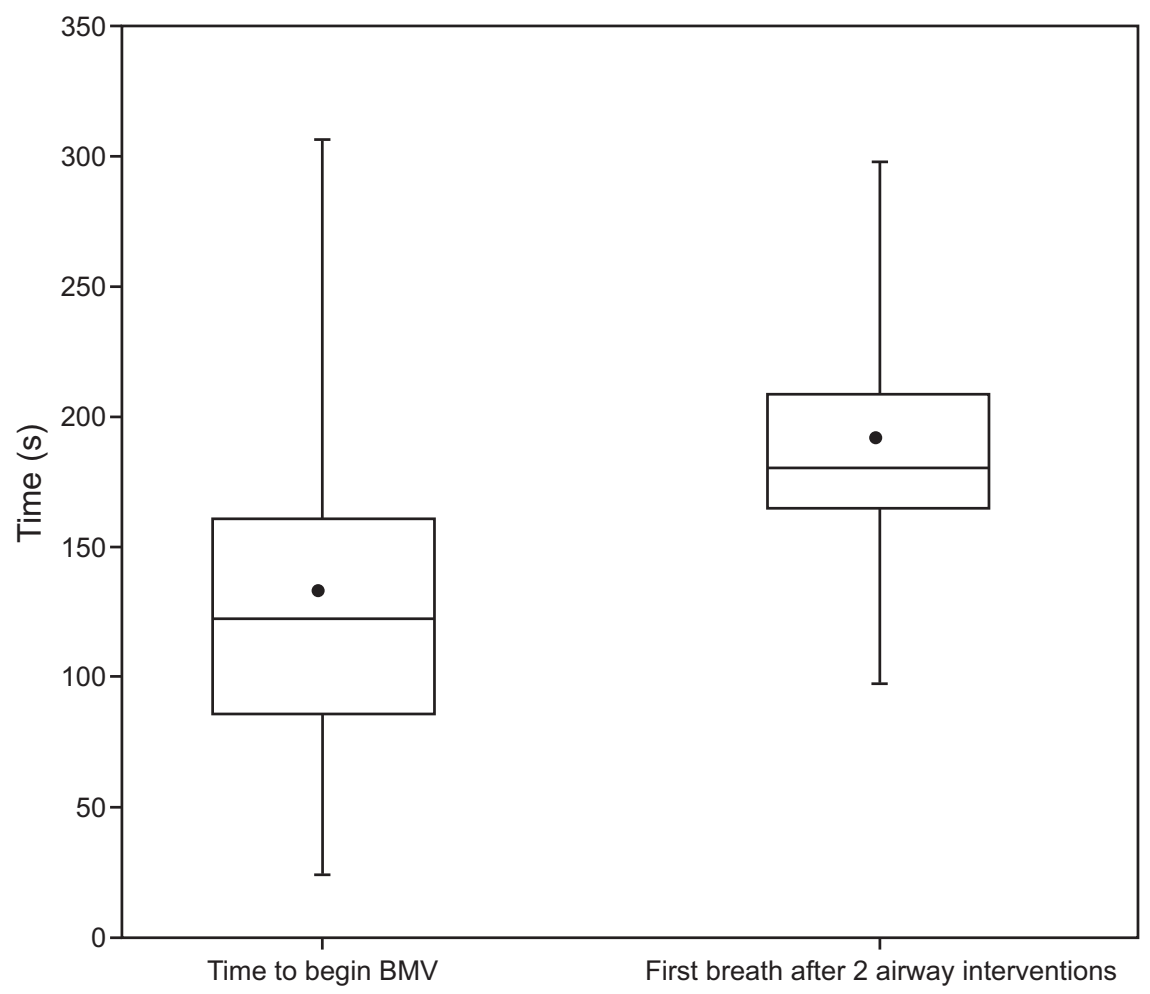

Fig. 2. Distribution of the time to bag-mask ventilation (BMV) intervention. 


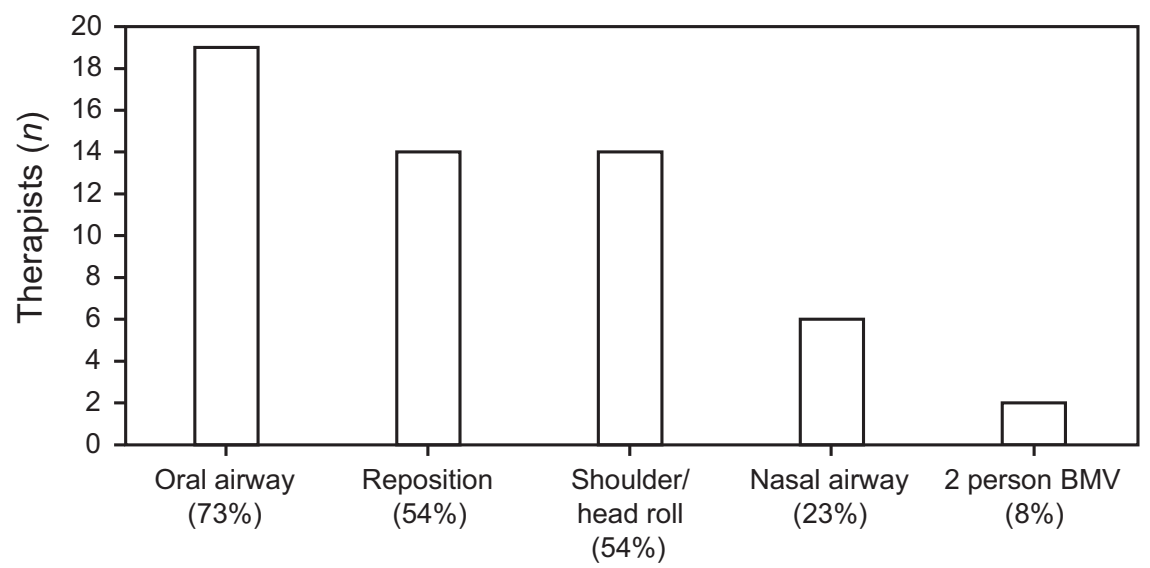

Fig. 3. Interventions used by respiratory therapists.

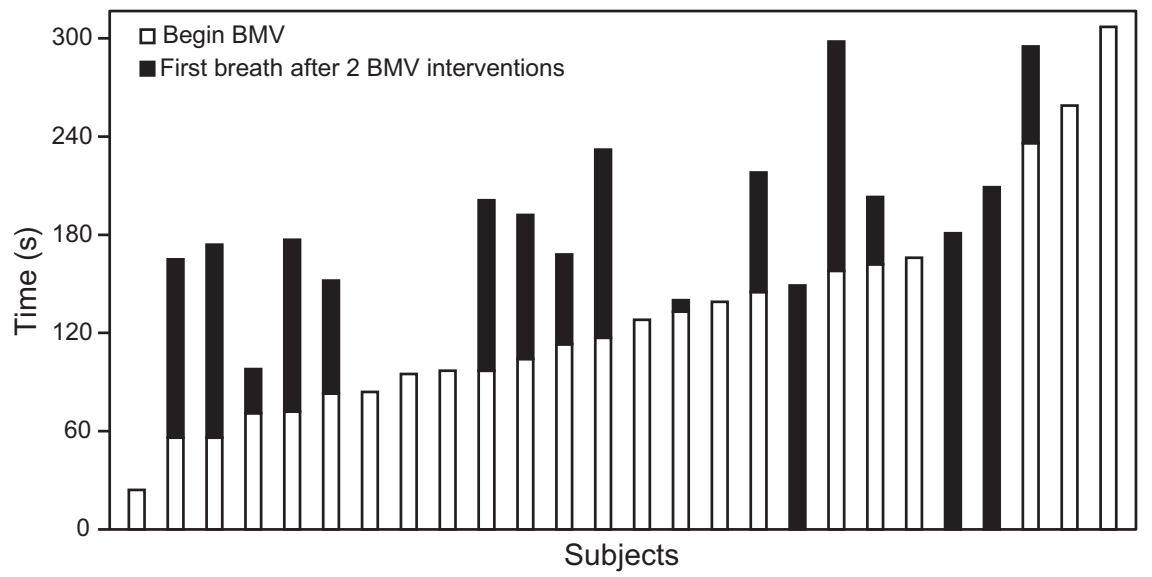

Fig. 4. Time to intervention for each respiratory therapist. BMV = bag-mask ventilation.

emergency medicine, our study focused on pediatric RTs'. Arguably, we could have expected our population to have a more-refined mental model of the management of difficult mask ventilation; however, this was not the case. Of note, in the article by Eppich et $\mathrm{al}^{8}$, they reported that their airway expert population of pediatric anesthesia and critical care attending physicians did have a clear and consistent mental model of what to do in the setting of difficult mask ventilation. Eppich et $\mathrm{al}^{8}$ also noted that trainees who had at least 1 month of anesthesia training had a clear mental model of difficult mask ventilation.

In our population, we were particularly surprised that only 2 of our participants (8\%) used 2-person bag-mask ventilation. Given that this technique can be performed immediately without having to wait for equipment as long as there is at least one other provider in close proximity, some airway experts argue to make this the first maneuver in the sequence of any standard approach to difficult mask ventilation. ${ }^{15,16}$ There was a strong preference for oral airway placement, which was surprising given its relative inaccessibility when compared with 2-person bag- mask ventilation and shoulder roll. In our experience, pointing out the rate-limiting steps and the reasons for a preferred sequence can help support building a strong mental model for learners. It is possible to teach a standard algorithm for management of difficult mask ventilation, even to novice providers, which can improve response time and predictability of performance. For example, our institution began to standardize how to teach adult difficult mask ventilation to novice airway providers. This included the following specific order of airway interventions during cardiac arrest: (1) reposition, (2) initiate 2-person bag-mask ventilation, (3) place oral airway, and (4) notify the team leader. Implementation of this training model with first-year medical students significantly improved their utilization of one or more of these interventions in a simulated patient without visible chest rise during bag-mask ventilation. ${ }^{14}$

Our results also revealed concerning deficits in fundamental airway skills of our pediatric RTs'. Although there is no established time frame to start bag-mask ventilation in a patient with impending respiratory failure, we considered that rapid initiation should be of top priority, partic- 


\section{RTs' Response Time to Simulated Difficult Bag-Mask Ventilation}

ularly for airway experts such as RTs'. We believed that $60 \mathrm{~s}$ was a reasonable time frame to assess patient respiratory effort, set up equipment, and attempt bag-mask ventilation. When we used this standard, only 3 of 26 of the pediatric RTs' (12\%) met the standard. In reviewing the videos, there were unnecessary interventions taken after recognition of respiratory distress that delayed bag-mask ventilation, which included requests for an additional patient history, thorough pulmonary auscultation, and manipulation of the bedside in preparation for extended bagmask ventilation (eg, such as adjusting side rails, lowering the head of the bed). Although these are all essential components of rapid response team performance, prioritizing them over time-sensitive, life-saving interventions can negatively impact a patient who is rapidly decompensating. Even those RTs' who had current PALS certification did not show any advanced skill in difficult mask ventilation management, which suggested that the available training was not sufficient to improve performance beyond what is currently available through clinical experience and our current hospital-based training.

As a result of these findings, adjustments were made to subsequent training to focus on improving the response time to those initial steps and on implementing a systematic algorithm for difficult mask ventilation airway maneuvers. Our goal outcome for training was to improve bedside care of pediatric subjects with impending respiratory failure. The components of the new standard difficult mask ventilation training with these focuses are listed as follows:

1. Rapidly evaluate respiratory effort and vital signs as the initial determinate of need for bag-mask ventilation.

2. Use action-linked phrases such as "the $\mathrm{S}_{\mathrm{pO}_{2}}$ is $<88 \%$ and the patient is bradypneic, I am starting mask ventilation."

3. Verbalize chest rise to the rapid-response team leader to foster a shared airway management cognition.

4. Focus on the use of 2-person bag-mask ventilation as the first difficult mask ventilation intervention because it is the most-effective method of bag-mask ventilation ${ }^{15,16}$ and usually available more quickly than adjunct airway devices.

5. After initiating 2-person bag-mask ventilation, anticipate the need for an oral airway by requesting it as the next step in case 2-person bag-mask ventilation is not successful. Share the location of the oral airway verbally.

We used the rapid-cycle deliberate practice as the method to teach future RT training sessions because it has been shown to improve pediatric team performance in respiratory and cardiac arrest management. ${ }^{14,17,19,20}$ The rapidcycle deliberate practice is a simulation-based instructional strategy that focuses on rapid acquisition of procedural and teamwork skills. It is an innovative pedagogic method that uses simulation as the instructional tool and incorporates components of deliberate practice, mastery learning, and crisis resource management to improve clinician performance during low-volume, high-risk, time-sensitive subjects events.

\section{Limitations}

There are a few potential limitations of the study that could have affected the results. It was possible that the therapists involved in the study were not able to adequately evaluate the simulated patient's respiratory distress due to limitations of the simulator. To minimize this effect, we chose an $\mathrm{S}_{\mathrm{pO}_{2}}$ value that was well below the reasonable threshold for initiating bag-mask ventilation, ${ }^{18}$ as well as a breathing frequency that was only $20-30 \%$ of the expected value for the simulated patient's age. These 2 items together were indicative of a need for assisted ventilation, regardless of the patient's observable respiratory distress. Another limitation was the potential for contamination of the subjects. The simulations occurred over a 3-month period, which provided an opportunity for the the RTs' to discuss their experiences and improve performance of subjects later in the data collection period.

In reviewing the data, there did not seem to be any trends toward better performance at the end of the year or any discernible patterns in selected adjunct interventions in later participants that would suggest prompting about correct performance. Another limitation was that participant success was measured by the use of an intervention, not the quality of the intervention. For example, the use of nasal airway or an oral airway was considered successful, although it was not clear enough in the video to ascertain whether the correct size pharyngeal airway was used. As a result, some actions may not have resulted in a positive clinical outcome, although they were considered successful in the video review. The sizing of airways was a debriefing topic to minimize the likelihood of negative learning during the simulation.

\section{Conclusions}

Analysis of the results of our study indicated that our team of pediatric RTs' did not share a standard method to approach impending respiratory failure. This included rapid recognition of the need for bag-mask ventilation and implementation of airway adjuncts. This may place children who are critically ill at risk of suboptimal management and threaten their clinical outcomes. Performance of the subjects indicated that no established cognitive framework, algorithm, or mental model had been effectively shared or taught, or, alternatively, that skill retention was poor. 


\section{RTs' Response Time to Simulated Difficult Bag-Mask Ventilation}

As a result of this video review, a change in the content and delivery method of bag-mask ventilation training was implemented to improve the time to performance of key interventions and to establish a clear framework for difficult mask ventilation management.

\section{ACKNOWLEDGMENTS}

The authors thank the staff of Johns Hopkins Medicine Simulation Center for their technical and operational support in the implementation of the simulation portion of this study.

\section{REFERENCES}

1. American Heart Association. Pediatric advances life support provider manual. Dallas, Texas: American Heart Association Inc; 2017.

2. Perretta JS. Making sense of caring for kids: A different approach to respiratory care. In: Perretta JS. Neonatal and pediatric respiratory care: a patient case method. Philadelphia, Pennsylvania: FA Davis; 2014;1-18.

3. Sunder RA, Haile DT, Farrell PT, Sharma A. Pediatric airway management: current practices and future directions. Pediatr Anesth 2012; 22(10):1008-1015.

4. Valois-Gómez T, Oofuvong M, Auer G, Coffin D, Loetwiriyakul W, Correa JA. Incidence of difficult bag-mask ventilation in children: A prospective observational study. Paediatr Anaesth 2013;23(10):920926.

5. Yildiz TS, Solak M, Toker K. The incidence and risk factors of difficult mask ventilation. J Anaesth 2005;19(1):7-11.

6. American Society of Anesthesiologists Task Force on Management of the Difficult Airway. Practice guidelines for management of the difficult airway: an updated report by the American Society of Anesthesiologists' task force on management of the difficult airway. Anesthesiology 2003;98(5):1269-1277.

7. El-Orbany M, Woehlck HJ. Difficult mask ventilation. Anesth Analg 2009;109(6):1870-1880.

8. Eppich WJ, Zonfrillo MR, Nelson KL, Hunt EA. Residents' mental model of bag-mask ventilation. Pediatr Emerg Care 2010;26(9):646652.
9. Barker TD, Schneider RE. Supplemental oxygenation and bag-mask ventilation. In: Walls RM, Murphy, Michael F, editors. Manual of emergency airway management. 3rd edition. Philadelphia, Pennsylvania: Lippincott Williams \& Wilkins; 2008:47-61.

10. Davies JD, Costa BK, Asciutto AJ. Approaches to manual ventilation. Respir Care 2014;59(6):810-822; discussion 822-824.

11. Hughes HK, Kahl LK. The Harriet Lane handbook. 21st edition. Philadelphia, Pennsylvania: Elsevier; 2017.

12. Bucher JT, Cooper JS. Bag mask ventilation. StatPearls Publishing LLC. 2018. Available at https://www.ncbi.nlm.nih.gov/books/ NBK441924/?report=printable. Accessed March 1, 2018.

13. Kory PD, Eisen LA, Adachi M, Ribaudo VA, Rosenthal ME, Mayo PH. Initial airway management skills of senior residents. Chest 2007; 132(6):1927-1931

14. Hunt EA, Duval-Arnould JM, Chime NO, Jones K, Rosen M, Hollingsworth $\mathrm{M}$, et al. Integration of in-hospital cardiac arrest contextual curriculum into a basic life support course: a randomized, controlled simulation study, Resuscitation 2017;114:127-132.

15. Davidovic L, LaCovey D, Pitetti RD. Comparison of 1-versus 2-person bag-valve-mask techniques for manikin ventilation of infants and children. Ann Emerg Med 2005;46(1):37-42.

16. Joffe AM, Hetzel S, Liew EC. A two-handed jaw-thrust technique is superior to the one-handed "EC-clamp" technique for mask ventilation in the apneic unconscious person. Anesthesiology 2010;113(4): 873-879.

17. Hunt EA, Duval-Arnould JM, Nelson-McMillan KL, Bradshaw JH, Diener-West M, Perretta JS, Shilkofski NA. Pediatric resident resuscitation skills improve after "Rapid Cycle Deliberate Practice" training. Resuscitation 2014;85(7):945-51.

18. Walls RM, Murphy MF. Manual of emergency airway management. 3rd ed. Philadelphia, Pennsylvania: Lippincot Williams \& Wilkins; 2008.

19. Brown KM, Mudd SS, Hunt EA, Perretta JS, Shilkofski NA, Diddle JW, et al. A multi-institutional simulation boot camp for pediatric cardiac critical care nurse practitioners. Pediatr Crit Care Med 2018; 19(6):564-571.

20. Sullivan NJ Duval-Arnould J, Twilley M, Smith SP, Aksamit D, Boone-Guercio P, et al. Simulation exercise to improve retention of cardiopulmonary resuscitation priorities for in-hospital cardiac arrests: a randomized controlled trial. Resuscitation 2015;86:6-13. 